\title{
Continuous-pole-expansion method to obtain spectra of electronic lattice models
}

\author{
Peter Staar, ${ }^{1}$ Bart Ydens, ${ }^{2}$ Anton Kozhevnikov, ${ }^{1}$ Jean-Pierre Locquet, ${ }^{2}$ and Thomas Schulthess ${ }^{1,3}$ \\ ${ }^{1}$ Institute for Theoretical Physics, ETH Zurich, 8093 Zurich, Switzerland \\ ${ }^{2}$ Laboratory of Solid-State Physics and Magnetism, KU Leuven, 3001 Leuven, Belgium \\ ${ }^{3}$ Swiss National Supercomputing Center, ETH Zurich, 6900 Lugano, Switzerland
}

(Received 14 November 2013; revised manuscript received 29 May 2014; published 11 June 2014)

\begin{abstract}
We present a new algorithm to analytically continue the self-energy of quantum many-body systems from Matsubara frequencies to the real axis. The method allows straightforward, unambiguous computation of electronic spectra for lattice models of strongly correlated systems from self-energy data that has been collected with state-of-the-art continuous time solvers within dynamical mean-field simulations. Using well-known analytical properties of the self-energy, the analytic continuation is cast into a constrained minimization problem that can be formulated as a quadratic programmable optimization with linear constraints. The algorithm is validated against exactly solvable finite-size problems, showing that all features of the spectral function near the Fermi level are very well reproduced and coarse features are reproduced for all energies. The method is applied to two well-known lattice problems, the two-dimensional Hubbard model at half filling, where the momentum dependence of the gap formation is studied, as well as a multiband model of $\mathrm{NiO}$, for which the spectral function can be directly compared to experiment. Agreement with published results is very good.
\end{abstract}

DOI: 10.1103/PhysRevB.89.245114

PACS number(s): 71.10.Fd, 71.15.Dx, 02.70.Hm, 05.30.-d

\section{INTRODUCTION}

Studies of electronic lattice models with intermediate to strong correlations have traditionally been very important in condensed matter physics. Most relevant models are not tractable with controlled analytic approximations in the parameter regions of interest, thus requiring numerical simulations for their solution. Currently, dynamical mean-field theory (DMFT) [1] is the method of choice in many investigations of this type of problems. For practical reasons DMFT approaches these models in imaginary rather than real time. Consequently, physical observables, such as spectral functions, can only be accessed indirectly via analytic continuation from the imaginary to the real axis.

Two methods are commonly used for analytic continuation to the real axis. In the Pade approximation [2], a fractional polynomial is fit to the data that has been computed on the Matsubara frequencies on the imaginary axis, and the polynomial is evaluated on the real axis. This procedure is rather general but for most physical cases requires fractional polynomials that violate known analytic properties of the fitted functions in the complex plane. The second and much more successful method is the maximum entropy method (MEM) [3-5]. This method analytically continues the imaginary time Green's function to the real frequency axis in order to obtain the spectrum $\mathcal{A}$. It is based on the relationship

$$
G(\tau)=\frac{-1}{\pi} \int d \omega \underbrace{\frac{e^{-\tau \omega}}{1+e^{-\beta \omega}}}_{=K(\omega, \tau)} \mathcal{A}(\omega) .
$$

A straightforward (numerical) inversion of Eq. (1) is impossible, since the spectrum at large frequencies $(\omega \gg 1)$ has only an exponentially small contribution to the imaginary time Green's function. Thus, at finite numerical precision, there are many different spectral functions that satisfy Eq. (1), since the kernel matrix $K(\omega, \tau)$ is singular. This problem was observed early on in the work of Schüttler and Scalapino [6,7]. The authors tried to solve Eq. (1) by minimizing the chi-squared value $\chi^{2}$ using a least-squared procedure:

$$
\chi^{2}=\sum_{i}\left[\tilde{G}\left(\tau_{i}\right)-\sum_{j} \mathcal{A}\left(\omega_{j}\right) K\left(\omega_{j}, \tau_{i}\right)\right]^{2} / \sigma_{i}^{2} .
$$

Here, $\tilde{G}\left(\tau_{i}\right)$ and $\sigma_{i}$ are, respectively, the measured Green's function and its standard deviation at imaginary time point $\tau_{i}$. The main problem with this approach is that the outcome is very sensitive to statistical noise on the measured Green's function, especially at large frequencies in the spectrum.

To overcome this problem, the maximum entropy method was developed. This method is based on the well-established Bayesian inference theory. The central idea in MEMs is to search for a spectral function that minimizes the chi-square value and maximizes the information entropy $\mathcal{S}$ [8], relative to a positive definite function $m(\omega)$, which has the correct high-frequency behavior:

$$
\mathcal{S}=-\int d \omega \mathcal{A}(\omega)-m(\omega)-\mathcal{A}(\omega) \log [\mathcal{A}(\omega) / m(\omega)] .
$$

Here the $m(\omega)$ function serves as the default model. In absence of the constraint in Eq. (1), maximizing the entropy will result in a spectrum $\mathcal{A}(\omega)$ equal to $m(\omega)$. For unknown nontrivial systems, finding a good default model might not be straightforward. Putting this issue aside, the MEM method finds the spectrum of the system by maximizing the likelihood function $F=\exp \left(-\chi^{2} / 2+\alpha \mathcal{S}\right)$. In the "classical" MEM, Bayesian logic is used to find the optimal value for the parameter $\alpha$. Consequently, there are no free parameters in the MEM, with the sole exception of the default model $m(\omega)$. Although the MEM has been extremely useful in many examples, it should be pointed out that there is no rigorous argument for using this particular likelihood function $F=$ $\exp \left(-\chi^{2} / 2+\alpha \mathcal{S}\right)$ as Sandvik points out [9]. The inclusion of the entropy term might lead to results, which are biased toward large entropy and thus smooth out features in the spectrum which are in fact present. 
With the introduction of continuous-time Monte Carlo solvers [10-14], it has become possible to directly measure the self-energy on the Matsubara axis with unprecedented speed [15,16] and accuracy [17]. This motivated us to investigate the possibility of an analytic continuation of the self-energy directly in frequency space with the relationship

$$
\Sigma(z)=\Sigma_{0}+\frac{1}{2 \pi} \int_{-\infty}^{\infty} d \omega \underbrace{\frac{1}{\omega-z}}_{=T(\omega, z)} \operatorname{Im}[\Sigma(\omega)] .
$$

As pointed out by Wang [18], performing the analytic continuation directly on the self-energy function instead of the Green's function is a good strategy to minimize the broadening effect of MEM. Furthermore, by keeping the analytic continuation in frequency space we can avoid the ill-defined high-frequency behavior caused by the exponential decay of the kernel in Eq. (1). This is easily demonstrated. On the Matsubara frequencies $\varpi_{m}=\pi T(1+2 m)$, the highfrequency part of the self-energy behaves like

$$
\Sigma\left(\varpi_{m} \gg 1\right) \approx \Sigma_{0}-\imath \frac{\Sigma_{1}}{\varpi_{m}}+\cdots, \quad \Sigma_{0}, \Sigma_{1} \in \mathbb{R} .
$$

Equating this to a high-frequency expansion of the righthand side of Eq. (4) gives

$$
\Sigma_{1}=\int_{-\infty}^{\infty} d \omega \operatorname{Im}[\Sigma(\omega)], \quad \operatorname{Im}[\Sigma(\omega)] \leqslant 0 .
$$

The imaginary part of the self-energy has a finite $L_{1}$-norm, since from standard field theory [19] it follows that the imaginary part of the self-energy is strictly negative on the real axis and thus must decay on the real axis for large frequencies. The exponential decay of the transfer function $K(\omega, \tau)$ in Eq. (1) for large frequencies is replaced by a polynomial decay of the new transfer function $T\left(\omega, \varpi_{m}\right)$ in Eq. (4), simply by keeping the analytic continuation completely in the frequency domain.

Despite the improvement to the ill-conditioned highfrequency problem on the real axis, inverting Eq. (4) remains nontrivial for a number of practical reasons. First, straightforward numerical inversion of the transfer-matrix $T\left(\omega, \varpi_{m}\right)$ is unstable. Second, in the typical case when the self-energy is obtained from a Monte-Carlo simulation, the algorithm has to be robust against statistical noise in the data-it should not extract information for the self-energy on the real axis from simple statistical noise. Finally, the constraint that the imaginary part of the self-energy has to be negative is difficult to enforce while solving a linear system.

For all these reasons we chose in the present paper to convert the inversion of Eq. (4) into a minimization problem. The desired self-energy is described by a functional form $f$ that is strictly positive on the real axis, and the minimization function $\Lambda$ is constructed from the L2-norm of the difference to the Monte Carlo simulation data on the Matsubara frequencies. The solution that minimizes $\Lambda$ corresponds to the solution of Eq. (4). Besides intrinsic robustness, the minimization approach has the advantage that strong constraints can easily be imposed on the targeted solution. The goal of this paper is to investigate the parametrization of the function $f$ and presenting a viable implementation of the constrained minimization problem.

A significant portion of the present work has been dedicated to the validation of the continuous pole expansion (CPE) algorithm. First we compare against spectra obtained via exact diagonalization (ED) solutions for isolated cluster models. With ED methods one computes the exact self-energy function on the imaginary and real axis for small problems, making a straightforward validation possible. Next we apply the CPE to self-energy data obtained for the single-band Hubbard model [20] in two dimensions (2D) at half filling, where the self-energy data is computed with the dynamical cluster approximation (DCA) [21-24] on a 128-site cluster. We validate against a formula commonly used in the literature to probe the spectral density at the Fermi energy. We illustrate how the CPE can be used to inspect the momentum dependence of the spectrum by investigating the momentum-dependent gap formation. This topic has been recently investigated on small clusters [25-27], but without any conclusions on the spectral functions. We will show that the CPE arrives at the same conclusions as in the literature and investigate the spectral functions more closely. Last, we apply the CPE to a multiband model of NiO, a well-studied material. We demonstrate how the orbital-dependent spectrum can be computed with the CPE and validate the results against experimental data available from the literature. As there are no exact results for the impurity problem, experimental x-ray photoemission (XPS), $\mathrm{X}$-ray emission spectroscopy, and Bremsstrahlung-isocromatspectroscopy (BIS) spectra are the next best option to validate the CPE algorithm on materials-specific models. We find a remarkably good agreement between theory and experiment.

The paper is structured as follows: In Sec. II we review some important analytical properties of Matsubara-Green's function and self-energy. In Sec. III we introduce the CPE for the selfenergy and treat thoroughly the numerical implementation. In Sec. IV, we compare the CPE with the exact results obtained with the ED solution of an isolated cluster. In Sec. V, we apply CPE to some physically relevant problems and compare the results of the CPE with the literature. Section VI contains the conclusions.

\section{ANALYTICAL PROPERTIES OF FERMIONIC GREEN'S FUNCTION AND SELF-ENERGY}

In order to motivate and later derive the continuous pole expansion (CPE) algorithm for the self-energy, we briefly review the analytical properties of the single particle Green's function as well as the self-energy for Fermionic systems. Following Abrikosov, Gorkov, and Dzyaloshinski [19], the single-particle propagator $G$ is defined as

$$
G(\vec{k}, \tau)=\left\langle T_{\tau}\left[c_{\vec{k}}^{\dagger}(\tau) c_{\vec{k}}(0)\right]\right\rangle,
$$

where the imaginary time $\tau \in[-\beta, \beta]$. Due to the timeordering operator $T_{\tau}$ and Fermionic commutation relations among the field operators $c^{\dagger}(\tau)$ and $c(\tau)$, where for simplicity we omit the momentum vector $\vec{k}$, the Green's function values for $\tau<0$ and $\tau>0$ are related by $G(\tau+\beta)=-G(\tau)$. Consequently, the Fourier transform of the Fermionic Green's function is only nonzero on the Matsubara frequencies 
$\varpi_{m}=\pi / \beta(2 m+1)$ with $m \in \mathbb{Z}$ and we have that

$$
G\left(\varpi_{m}\right)=\int_{0}^{\beta} d \tau e^{i \varpi_{m} \tau} G(\tau) .
$$

Since there exists a unique, analytical function that coincides with the infinite sequence $\left\{l \varpi_{m}, G\left(\varpi_{m}\right)\right\}$ in the complex plane,[28] we define the Green's function $G(z)$ on the entire complex plane as the unique analytical continuation of this infinite sequence. Furthermore, a straightforward expansion of Eq. (7) in terms of the eigenenergies and eigenbasis of the system reveal that there exists a positive, integrable, real function $\rho(\omega)$ such that the Green's function on the real axis can be obtained as

$$
G\left(\varpi_{m}\right)=\int_{-\infty}^{+\infty} d \omega^{\prime} \frac{\rho\left(\omega^{\prime}\right)}{\omega^{\prime}-\varpi_{m}} .
$$

Due to uniqueness we can generalize Eq. (9) to anywhere in the (upper) complex plane and obtain

$$
G(z)=\int_{-\infty}^{+\infty} d \omega^{\prime} \frac{\rho\left(\omega^{\prime}\right)}{\omega^{\prime}-z} .
$$

Since $\rho$ is a real and positive function, we can deduce from Eq. (10) that the following analytical properties hold for the Green's function:

$$
\begin{aligned}
\operatorname{Im}[G(\omega+i \varpi)] & <0 \text { if } \varpi>0 \\
G^{I}(i \varpi) & =\overline{G^{I}(-i \varpi)} .
\end{aligned}
$$

It follows that the single-particle propagator can only have complex zeros on the real axis.

The self-energy $\Sigma$ is related to the Green's function via the Dyson equation:

$$
\Sigma(z)=G_{0}^{-1}(z)-G^{-1}(z) .
$$

Since $G(z)$ and $G_{0}(z)$ have no zeros in the upper complex plane, it follows that their inversion cannot introduce poles in the upper plane of the self-energy. Hence, the self-energy is also analytic in the upper complex plane, with the possible exception on the real axis. The absence of poles in the upper complex plane permits use of the residue theorem

$$
\Sigma(z)=\frac{1}{2 \pi \imath} \lim _{\delta \rightarrow 0^{+}} \int_{-\infty}^{+\infty} d \omega \frac{\Sigma(\omega+\imath \delta)}{\omega-z} .
$$

Due to causality, the self-energy must be negative everywhere in the upper complex plane. This combined with the Kramers-Kronig relationships results in the identity

$$
\begin{aligned}
& \Sigma(z)=\frac{1}{2 \pi} \lim _{\delta \rightarrow 0^{+}} \int_{-\infty}^{+\infty} d \omega \frac{\operatorname{Im}[\Sigma(\omega+\imath \delta)]}{\omega-z}, \\
& \lim _{\delta \rightarrow 0^{+}} \operatorname{Im}[\Sigma(\omega+\imath \delta)]<0 .
\end{aligned}
$$

\section{CONTINUOUS POLE EXPANSION FOR THE SELF-ENERGY}

In the first part of this section, we present how the CPE implements the analytical continuation of the complex self-energy function from the Matsubara frequencies on the imaginary axis to the real axis. The implementation assumes that the stochastic noise from the Quantum Monte Carlo integration is small compared to the value of the function. Since the influence of the stochastic noise on the final solution is hard to judge, we propose in the second part of this section a procedure that measures the robustness of our solution versus the stochastic noise on the complex self-energy function. In this way, we do not only provide a solution to the analytical continuation problem but also a method of operation to test the reliability of the solution found by the CPE.

\section{A. Implementation of the CPE}

Motivated by Eq. (14), the self-energy on the Matsubara frequencies can be parametrized with a positive, real function $f$,

$$
\tilde{\Sigma}\left(\varpi_{m}\right)=\Sigma_{0}+\int_{-\infty}^{+\infty} d \omega \frac{f(\omega)}{l \varpi_{m}-\omega},
$$

with

$$
f \geqslant 0, \Sigma_{0} \in \mathbb{R}, \quad \int_{\infty}^{\infty} d x f(x)=\Sigma_{1} .
$$

The aim of the CPE algorithm is to search for the positive function $f$, which minimizes the norm $\Lambda$ :

$$
\Lambda(f)=\sum_{m=0}^{M}\left|\tilde{\Sigma}\left(\varpi_{m}\right)-\Sigma\left(\varpi_{m}\right]\right|^{2} .
$$

In order to find $f$, we will decompose it in the basis of a regular-spaced, piecewise linear function. If $\left\{\omega_{n}=n \Delta / N\right\}$ (with $n \in\{-N, \ldots, N\}$ and $\Delta$ denoting the minimum and maximum range for a nonzero self-energy) forms our regularspaced grid on the real axis, we can define the decomposition explicitly with the help of the step-function $\theta$ :

$$
\begin{aligned}
f(\omega)= & \sum_{n=-N}^{N} \alpha_{n} \phi_{n}(\omega) \quad \text { with } \alpha_{n} \geqslant 0, \\
\phi_{n}(\omega)= & \theta\left(\omega-\omega_{n-1}\right) \theta\left(\omega_{n}-\omega\right) \frac{\omega-\omega_{n-1}}{\omega_{n}-\omega_{n-1}} \\
& +\theta\left(\omega-\omega_{n}\right) \theta\left(\omega_{n+1}-\omega\right) \frac{\omega_{n+1}-\omega}{\omega_{n+1}-\omega_{n}} .
\end{aligned}
$$

It should be noted that for large enough $N$, any continuous function on the real axis in the range of $[-\Delta, \Delta]$ can be approximated arbitrarily well using the basis set $\phi_{n}(\omega)$. As a consequence, the particular choice of this basis set is not a strong constraint for the validity of the CPE. However, this particular basis function is extremely handy for enforcing the positivity of the function $f$, since $f$ will be positive if and only if all coefficients $\alpha_{n}$ are positive. With this explicit decomposition in Eq. (17), we can perform the integral in Eq. (15) analytically and rewrite $\tilde{\Sigma}(z)$ into a much simpler form:

$$
\begin{aligned}
\tilde{\Sigma}(z)= & \Sigma_{0}+\sum_{n=-N}^{N} \Phi_{n}(z) \alpha_{n} \quad \text { with } \alpha_{n} \geqslant 0, \\
\Phi_{n}(z)= & \frac{\omega_{n-1}-z}{\omega_{n-1}-\omega_{n}} \log \left(\frac{\omega_{n-1}-z}{\omega_{n}-z}\right) \\
& -\frac{\omega_{n+1}-z}{\omega_{n}-\omega_{n+1}} \log \left(\frac{z-\omega_{n}}{z-\omega_{n+1}}\right) .
\end{aligned}
$$

Next we define a transfer matrix $A_{m, n}=\Phi_{n}\left(i \varpi_{m}\right)$ and rewrite the norm $\Lambda$ into a least square problem with boundary 
conditions:

$$
\Lambda=\sum_{m=0}^{M}\left|\Sigma_{0}+\sum_{n=-N}^{N} A_{m, n} \alpha_{n}-\Sigma\left(\varpi_{m}\right)\right|^{2}, \quad \alpha_{n} \geqslant 0 .
$$

By expanding the norm and gathering the terms of the same order in $\alpha_{n}$, one obtains an explicit quadratic form for the norm $\Lambda$ as a function of the constant transfer matrix $A_{m, n}$ and the coefficients $\Sigma_{0}$ and $\left\{\alpha_{n}\right\}$ :

$$
\begin{aligned}
\Lambda & =M \Sigma_{0}^{2}-2 \Sigma_{0} \sum_{m=0}^{M-1} \operatorname{Re}\left[\Sigma\left(i \varpi_{m}\right)\right]+\vec{\alpha} Q \vec{\alpha}^{T}+\vec{q} \vec{\alpha}^{T}+C, \\
Q & =\left(\operatorname{Im}[A]^{T} \operatorname{Im}[A]+\operatorname{Re}[A]^{T} \operatorname{Re}[A]\right) \\
\vec{q} & =2\left(\left(\Sigma_{0}-\operatorname{Re}[\vec{\Sigma}]\right) \operatorname{Re}[A]-\operatorname{Im}[\vec{\Sigma}] \operatorname{Im}[A]\right) \\
\vec{\alpha} & =\left\{\alpha_{-N}, \ldots, \alpha_{N}\right\} \text { and } \\
\vec{\Sigma} & =\left\{\Sigma\left(i \varpi_{0}\right), \ldots, \Sigma\left(i \varpi_{m}\right)\right\} .
\end{aligned}
$$

The CPE algorithm thus rephrases the problem of analytic continuation of a noisy function to a quadratic programmable optimization problem, with linear constraints. These types of problems are well known, and many algorithms exists to find the minimum. From Eqs. (19), it follows that $Q$ is positive definite in the parametrization space $\alpha_{n} \geqslant 0$, and according to Eq. (16), $\Lambda$ has a trivial lower bound. In a quadratic programmable optimization problem, these conditions are sufficient to guarantee a unique solution for which our norm $\Lambda$ is minimized. Consequently, given $\Delta, N$, and $M$, there is a unique set of $\left\{\alpha_{n}\right\}$ and $\Sigma_{0}$ that minimize the norm $\Lambda$. The Frank-Wolfe algorithm [29] (FWA) is the simplest method for solving a quadratic programmable optimization problem. It is applied in the context of the CPE algorithm in the following way: First, we subtract the zeroth moment $\Sigma_{0}$ of the measured self-energy $\Sigma$. This ensures integrability of $\Sigma$ and $\tilde{\Sigma}$ along the real axis. Next, we choose an initial set of $\left\{\alpha_{n}\right\}$ and compute the gradient of $\Lambda$ toward $\left\{\alpha_{n}\right\}$ and $\Sigma_{0}$ :

$$
\begin{aligned}
\vec{\nabla}_{\{\alpha\}} \Lambda= & 2 \operatorname{Im}[A]^{T}(\operatorname{Im}[A] \vec{\alpha}-\operatorname{Im}[\vec{\Sigma}]) \\
& +2 \operatorname{Re}[A]^{T}\left(\operatorname{Re}[A] \vec{\alpha}+\Sigma_{0}-\operatorname{Re}[\vec{\Sigma}]\right) . \\
\frac{\partial \Lambda}{\partial \Sigma_{0}}= & 2 \sum_{m=0}^{M-1}\left(\operatorname{Re}[A] \vec{\alpha}+\Sigma_{0}-\operatorname{Re}\left[\Sigma\left(i \varpi_{m}\right)\right]\right) .
\end{aligned}
$$

Notice that the initial guess of $\left\{\alpha_{n}\right\}$ is unimportant, since there is only 1 minimum in our convex search space. We now search a $\lambda$ that minimizes the norm $\Lambda$ along the direction of $-\vec{\nabla}_{\left\{\alpha_{n}\right\}} \Lambda$. Special care has to be taken to enforce positivity of all coefficients $\left\{\alpha_{n}\right\}$. This is accomplished by point-wise application of the $\rho$ ramp function [30]:

$$
\begin{aligned}
& \tilde{\Sigma}(\lambda)=A \rho\left(\vec{\alpha}-\lambda \vec{\nabla}_{\{\alpha\}} \Lambda\right) \\
& \Lambda(\lambda)=\sum_{m=0}^{M}(\operatorname{Im}[\tilde{\Sigma}(\lambda)-\vec{\Sigma}])^{2}+(\operatorname{Re}[\tilde{\Sigma}(\lambda)-\vec{\Sigma}])^{2} .
\end{aligned}
$$

The parameter $\lambda_{\min }$ that minimizes our norm can now be used to generate a new set of coefficients $\{\alpha\}$ :

$$
\vec{a}_{i+1}=\rho\left(\vec{\alpha}_{i}-\lambda_{\min } \vec{\nabla}_{\left\{\alpha_{i}\right\}} \Lambda\right) .
$$

We continue this iterative process until $\Lambda$ is numerically converged to a minimum value.

This minimization approach of the CPE algorithm has several major benefits compared to other algorithms. First, the CPE algorithm depends only on three external parameters, $\Delta, N$, and $M$, as we do not consider the to-be-fitted self-energy points on the imaginary axis as separate degrees of freedom. Furthermore, for these three parameters, there is a unique solution in our allowed search space, since the norm can be rewritten as a quadratic function with a positive definite kernelmatrix $A$. Second, CPE is numerically stable against noise on the measured self-energy $\Sigma$, since we use a constrained fitting procedure. This robustness is important in the DMFT context, where the self-energy is computed via a stochastic process. Third, CPE is a self-consistent method, which returns a measure for the quality of the fit via $\Lambda$. This measure can be used to adjust the external parameters in the case of a bad fit.

In all the cases we have considered in this paper, the typical value of three parameters, $\Delta, N$, and $M$, were, respectively, 20, 4096, and 64. We observed insignificant changes in the spectra when the parameters were doubled and thus consider it converged. This robustness of the results versus these parameters is not surprising, since it simply reflects the robustness of the constrained, minimization procedure.

\section{B. Propagation of the stochastic error}

In this subsection, we want to discuss how the stochastic noise on the self-energy function might influence the final solution given by the CPE and how to quantify this influence. Since the CPE is geared toward finding the spectrum of interacting lattice models that are solved with the quantum Monte Carlo method, it is imperative to have a simple, straightforward estimator of the error on our analytically continued functions. The central idea of our approach is to generate a set self-energy function, by adding a different artificial, Gaussian noise signal to the measured self-energy function on the Matsubara frequencies. Here, the amplitude of the Gaussian noise is equal to error on the measured self-energy function, which originates from the quantum Monte Carlo solver. For each of these self-energies in the set, we can then perform a CPE analytic continuation and thus obtain a set of self-energy functions on the real axis. For each of these self-energy functions, we can compute the Green's function on the real axis straightforwardly and thus arrive at an ensemble of slightly different spectra. One can then compute the mean and standard deviation of the spectrum at each frequency. If the solutions of the CPE are highly susceptible to the stochastic noise, one would expect to observe a large variation on its solutions and thus observe a large standard deviation for the analytically continued self-energy functions, Green's functions, and spectra.

\section{VALIDATION OF CPE WITH EXACT DIAGONALIZATION}

Our first validation step is to compare the CPE algorithm to ED results for exactly solvable models. Without too much difficulty, we can presently solve the single-band Hubbard model on an isolated eight-site cluster. Due to the finite size 

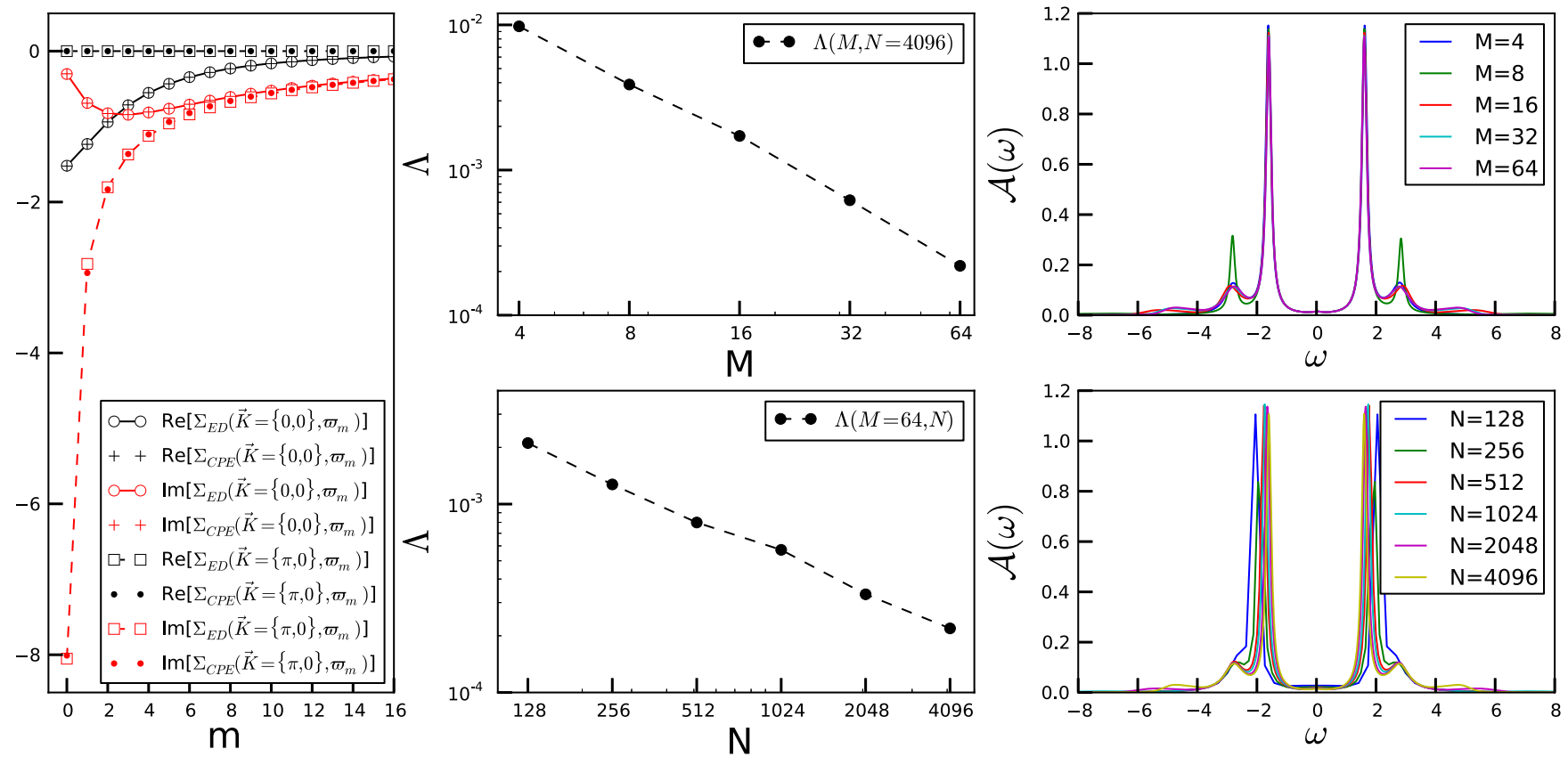

FIG. 1. (Color online) On the left, we compare the self-energy obtained from ED on the Matsubara frequencies with the fitted self-energy from the CPE for two different $\vec{K}$ points. We observe that the CPE provides a very good fit. In the middle, we show the evolution of the $\Lambda$ norm versus the internal parameters $M$ and $N$. We can observe a steady decay of the norm versus both parameters. On the right, we show the corresponding spectra obtained with these parameters. We can see that the spectra converge for $M \geqslant 32$ and $N \geqslant 1024$.

of the system, the Hamiltonian has a limited and manageable number of terms and is represented as

$$
\begin{aligned}
\mathcal{H}= & -t \sum_{\sigma=\uparrow, \downarrow} \sum_{\langle i, j\rangle=1}^{8} c_{i, \sigma}^{\dagger} c_{j, \sigma} \\
& +\frac{U}{2} \sum_{\sigma=\uparrow, \downarrow} \sum_{i=1}^{8}\left(n_{i, \sigma}-1 / 2\right)\left(n_{j,-\sigma}-1 / 2\right) .
\end{aligned}
$$

The Hamiltonian acts on a Fock space, composed of $2^{16}=$ 65536 states. After applying total number and magnetization symmetries, the matrix can be block-diagonalized with a maximum block size of 4900. Using standard eigenvalue decomposition routines of LAPACK [31], we can obtain all the eigenenergies $\left\{\epsilon_{i}\right\}$ and eigenstates $\left\{\left|\Psi_{i}\right\rangle\right\}$ of the isolated eight-site cluster. Following standard many-body theory, we can now compute the Green's function anywhere in the (upper) complex plane:

$$
G(\nu, \mu, z)=\sum_{i, j} \frac{e^{-\beta \epsilon_{i}}}{\mathcal{Z}} \frac{\left\langle\Psi_{i}\left|c_{\nu}\right| \Psi_{j}\right\rangle\left\langle\Psi_{j}\left|c_{\mu}^{\dagger}\right| \Psi_{i}\right\rangle}{z-\epsilon_{i}+\epsilon_{j}} .
$$

Here, the symbols $\nu$ and $\mu$ are shorthand notations for the band, spin, and cluster $K$ point $v=\left\{b_{v}, s_{v}, \vec{K}_{v}\right\}$. By solving the cluster twice, once with and once without the interaction, we can obtain, respectively, the interacting and noninteracting Green's function. From these two functions, we can obtain the self-energy in momentum space through the Dyson's equation anywhere in the complex plane:

$$
\Sigma(\vec{K}, z)=G_{0}^{-1}(\vec{K}, z)-G^{-1}(\vec{K}, z) .
$$

The idea is now to evaluate the self-energy on the real axis and on the Matsubara frequencies that are located on the imaginary axis. In this way, we can use the self-energy on the Matsubara frequencies as an input for the CPE, and compare the analytically continued self-energy with the exact result.

In this section, we will consider the Hubbard model with parameters $U=4, t=1$, and $\beta=10$. The first step of the CPE method consists of finding the parameter ranges for $M$ and $N$, where the CPE spectra are converged. In Fig. 1, we show the evolution of the $\Lambda$ norm versus the internal parameters $M$ and $N$ on the left side. Since the self-energy is obtained with $\mathrm{ED}$, we do not have any numerical noise and thus observe a steady decline of the norm versus both parameters. On the righthand side, we plot the resulting spectra for each parameter set $(M, N)$. We can clearly see that the spectra converge for $M \geqslant 16$ and $N \geqslant 1024$, as expected. The convergence of the spectra versus $M$ is very fast, because the essential information of the spectrum is captured by the self-energy function of the lowest Matsubara frequencies. Hence, once the tail section is captured relatively well, the spectra will not change anymore by increasing $M$. In order to converge the spectra versus $N$, we need to go to much larger values, mainly in order to capture the strong divergency of the self-energy around $\omega=0$, as can be seen in Fig. 2. However, once the intervals $\Delta / N$ are small enough such that our piecewise linear basis functions $\phi_{n}$ can describe the peak accurately, we observe that the the norm and spectra converge fast.

Once the parameters $M$ and $N$ are determined that result in a convergent of the CPE spectrum, we can start to compare the CPE results with the exact results from ED. In Fig. 2, we show the self-energy for various $\vec{K}$ points on the real axis with an offset of $\delta=0.1$. By comparing the ED results with the analytically continued self-energy, we can study the strengths and weaknesses of the CPE. Looking at the imaginary part of the self-energy, we observe that the high-frequency behavior 

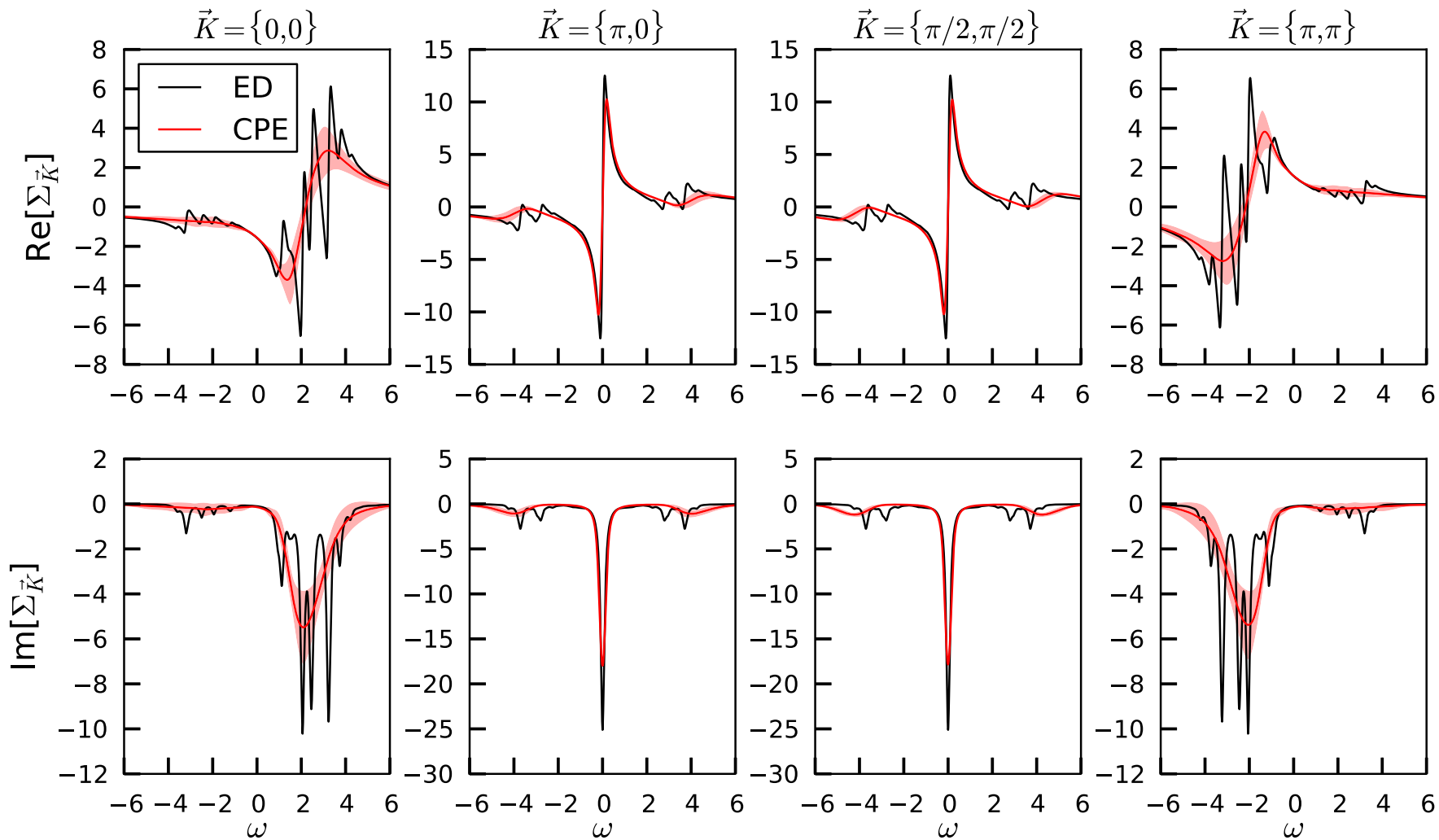

FIG. 2. (Color online) Comparison of the real and imaginary part of the self-energy $\Sigma(\vec{K}, \omega)$ obtained with ED as well as with CPE. We observe that the CPE can capture the self-energy remarkably well around the Fermi energy $(\omega=0)$, as well as the broad features far away from it. Furthermore, the standard deviation (pink band) is the largest in the region where the CPE makes consistent errors due to the unsmooth self-energy (e.g., for $\vec{K}=\{0,0\}$ and $\omega \in[0,5])$.

of the self-energy decays smoothly and does not diverge. As a consequence, we do not need to introduce a default function $m(\omega)$ like in the MEM in order to control the behavior of the analytically continued function at large energies. Furthermore, we see that the CPE captures remarkably well the self-energy around the Fermi energy $(\omega=0)$, as well as the broad features far away from it. Sharp features are, however, not well reproduced. This is clear from the imaginary part of the self-energy at $\vec{K}=\{\pi, 0\}$. The divergence at the Fermi energy is underestimated and the features at the interval $\omega=[-4,-2]$ are absent in the CPE self-energy.

By introducing artificial Gaussian noise with standard deviation of $1 \%$ of the maximum absolute value of the self-energy, we can investigate how robust the CPE method is versus stochastic noise, which is inevitably present in QMC data. As outlined in Sec. III B, we can compute the standard deviation of multiple analytically continued self-energy functions, each originating from the same ED Matsubara self-energy but with a different Gaussian noise signal added. The standard deviation of the analytically continued self-energy functions is represented by the pink band surrounding the average CPE result. One can clearly see that the band is the largest in the region where the $\mathrm{CPE}$ does not capture the ED self-energy very well due to the many poles, which lead to a very unsmooth self-energy function. Consequently, we can conclude that one should not expect to be able to determine sharp features in the spectrum with the CPE far from the Fermi surface. Only broad features are captured accurately far from the Fermi surface. On the other hand, you should be able to determine the accuracy of the CPE by doing the propagation of the stochastic error properly as outlined in Sec. III B.

In Fig. 3, we compare the spectra obtained with MEM and CPE to the spectrum of ED. This allows us to showcase the strengths and weaknesses of the CPE and the well-established MEM versus the exact spectrum. Since we are considering

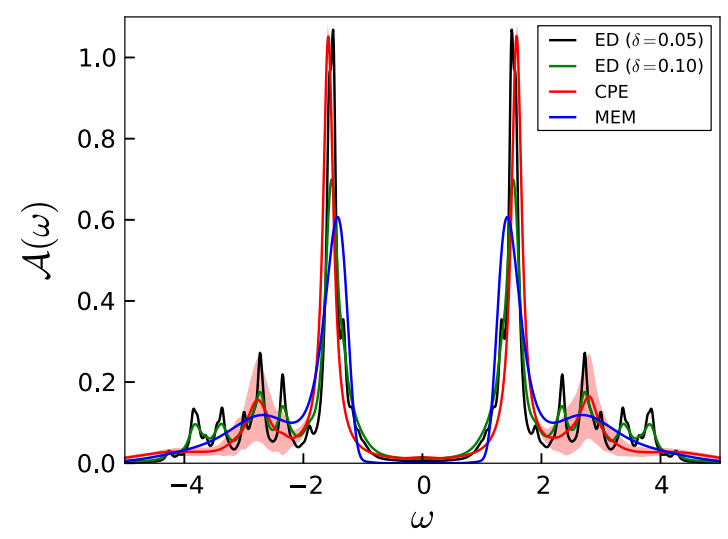

FIG. 3. (Color online) Comparison of the spectrum obtained with ED for two different offsets $i \delta$ versus the spectrum obtained with the $\mathrm{CPE}$ and the MEM. Both approximate methods seem to capture the width of the gap as well as the broad Hubbard bands at $\omega \approx \pm 3$ very well. Furthermore, the standard deviation (pink band) is the largest in the region where the $\mathrm{CPE}$ makes the maximum error. 
a finite system, we know that there are a finite number of poles in the spectrum, each with a finite weight. In order to obtain a smooth spectrum, we need to plot the spectrum with an offset $i \delta$ above the real axis. To show the influence of the offset, we have shown the ED spectrum with two different offsets, i.e., $\delta=0.05$ and $\delta=0.1$. The offset clearly does not change the position of the peaks but only the height and width. Consequently, one should focus on the position of the peaks rather than the height when comparing different spectra. In Fig. 3, one can clearly observe that the gap around the Fermi surface is well represented by the CPE and the MEM, as well as the broad Hubbard bands at $\omega \approx \pm 3$. However, the broad bands around $\omega \approx \pm 3$ are much more pronounced in the CPE and, therefore, the CPE seems to capture the physics slightly better. The standard deviation on the CPE result in this region is also large, which seems to suggest that the CPE is aware that it only has an approximate solution in this region.

Before we start to discuss the results of the CPE on lattice problems, we should point out that the spectrum of lattice models is generally smoother than that of finite-size clusters, since they have an infinite number of eigenvalues with an infinitesimal weight instead of a finite set of eigenvalues. The finite set of eigenvalues introduces poles with a finite weight on the real axis and thus gives rise to the sharp features observed in Figs. 2 and 3. Consequently, we expect that the CPE should perform better for lattice models than for the finite-size model we just considered.

\section{APPLICATION TO LATTICE PROBLEMS}

We now apply the CPE algorithm to two well-known lattice problems, in order to demonstrate the ability of the algorithm to reproduce published numerical or experimental results. We will consider two fundamentally different problems. First we use the CPE to investigate the momentum dependence of the spectrum in the single-band Hubbard model in two dimensions at half-filling. Particularly the momentum dependence of the gap formation has received a lot of interest recently [25,27], and there are several results in the literature to compare to. We can further validate the CPE by looking at the $\vec{K}$-dependent self-energy obtained from quantum Monte Carlo simulations within the DCA. Second, we will use the CPE to compute the spectrum of $\mathrm{NiO}$ and compare the latter to experimental data. The prediction of a $4.3 \mathrm{eV}$ gap around the Fermi energy is one of the great successes of LDA+DMFT and consequently has to be reproduced by CPE-based spectral functions. We will show that this is the case, and that in combination with LDA+DMFT, the CPE can be seen as a practical and unambiguous tool to compute spectral functions of real materials that compare rather well with experiments.

\section{A. Momentum-dependent gap formation in half-filled 2D Hubbard model}

The combination of the dynamical cluster approximation together with the CPE algorithm allows us to investigate the momentum dependency of the spectrum in different regimes of the phase diagram. Recently, much attention has been given to the momentum-dependent gap formation at half-filling in the single-band Hubbard model. In particular, it has been shown [25,27] that for a specific interaction strength of $U / t=6$, the antinodal regions $([ \pm \pi, 0]$ and $[0, \pm \pi])$ lose their spectral weight contribution at the Fermi energy faster than the nodal regions $([ \pm \pi / 2, \pm \pi / 2])$. This momentum anisotropy in the self-energy is a very interesting phenomenon since exotic ground states, such as the antiferromagnetic and $d$-wave superconducting state require a momentum-dependent self-energy.

Here we investigate this momentum anisotropy on a 128site cluster at half-filling for an interaction strength of $U=$ 6 and hopping amplitude $t=1$. A 128-site cluster is large enough to allow a careful study of the momentum anisotropy along the Fermi surface and will give us an intimate view on the gap formation at zero doping. In the literature, the momentum anisotropy of the gap formation has been investigated by using the identity

$$
\begin{aligned}
\beta G(\vec{k}, \tau=\beta / 2) & =\beta / 2 \int d \omega \frac{\mathcal{A}(\vec{k}, \omega)}{\cosh (\beta \omega / 2)} \\
& \stackrel{\beta}{ } \underset{ }{\approx} \pi \mathcal{A}(\vec{k}, \omega=0) .
\end{aligned}
$$

At low temperatures, the quantity $\beta G(\vec{k}, \tau=\beta / 2)$ provides a good estimate for the spectrum at the Fermi energy, since the function $1 / \cosh (\beta \omega / 2)$ becomes a $\delta$ function for $\beta$ going to infinity. Since the imaginary time function can be computed directly with a quantum Monte Carlo solver, the spectrum at the Fermi energy can be probed straightforwardly, without the need to do an analytical continuation of the self energy or Green's function. With the CPE, we can obtain the spectrum $\mathcal{A}(\vec{K}, \omega)$ on the entire real axis. We can thus verify the accuracy of the CPE by performing the integral on the righthand side of Eq. (25) and comparing to the quantity $\beta G(\vec{k}, \tau=\beta / 2)$.

The crosses in Fig. 4 show for various $\vec{k}$ points on the righthand side of Eq. (25). The open circles are the results for the corresponding lefthand side of the equation, where the integral was computed with the CPE (note that equality is not enforced during the minimization process). The agreement is very good and implies that all features we compute with the CPE algorithm at or near the Fermi energy will be in agreement with results in the literature that have been computed with the lefthand side of Eq. (25).

The Mott transition at zero percent doping has been intensively investigated since the first DMFT results became available [1,24]. The behavior of the spectrum as a function of the temperature is therefore well known in this region and offers a benchmark for the CPE algorithm. In Fig. 5, we show the temperature dependence of the spectrum $\mathcal{A}(\omega)$. As expected, the spectrum drops quickly in a broad region around the Fermi energy $(\omega=0)$ in order to form a gap. At the same time, two broad Hubbard bands emerge at $\omega= \pm 4$ and two sharp peaks emerge at the edge of the gap. These sharp features originate from the van Hove singularities, which typically go together with the formation of a gap. The advantage of analytically continuing the self-energy as opposed to the Green's function now becomes clear. Since the CPE reliably reproduces broad features it is perfectly well suited for analytic continuation of irreducible quantities like the self-energy, which are assumed to be rather smooth. The sharper features such as the van Hove singularities will then 


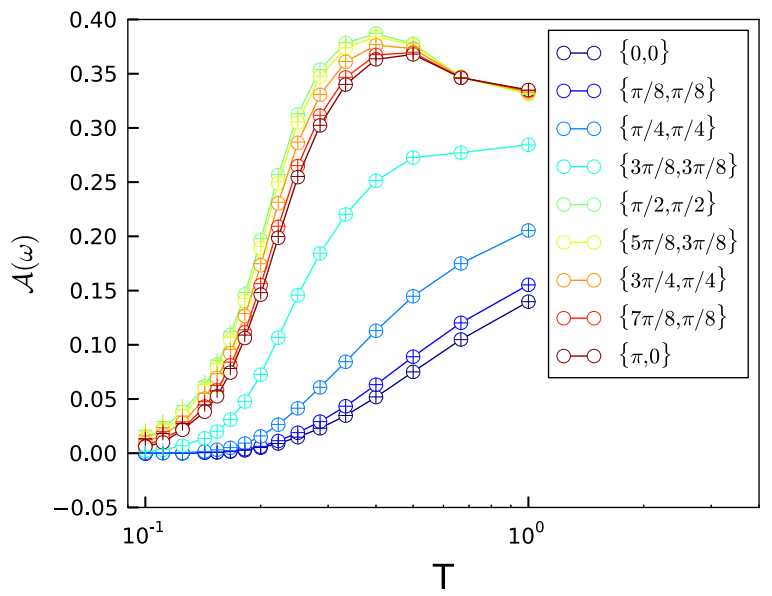

FIG. 4. (Color online) Verification of the CPE algorithm through Eq. (25) at zero percent doping. The lefthand side $\beta G(\vec{k}, \beta / 2)$ is depicted by the connected open circles, while the righthand side is given by the crosses. Given that this relationship is not enforced during the minimization process, the agreement is remarkably good. Only at low temperatures, we see a small discrepancy between the circles and the crosses for the $k$ points on the Fermi surface, due to the sharp nature of the van Hove singularities in the spectrum (see also Fig. 6).

be generated by the Brillouin zone integration of the Green's function on the real axis:

$$
\mathcal{A}(\omega)=-\frac{1}{\pi} \int d \vec{k} \operatorname{Im}\left[\frac{1}{\omega+\imath \delta+\mu-\epsilon(\tilde{\mathrm{k}})-\Sigma(\tilde{\mathrm{K}}, \omega)}\right] .
$$

Here, we used a common offset of $\delta=0.1$. The Brillouin zone integration is performed using the tetrahedron integration method (TIM) [32,33]. The TIM was developed especially to handle integrals over inverse functions. The inversion introduces poles in the integrand, and TIM can treat these poles in a numerically controlled way.

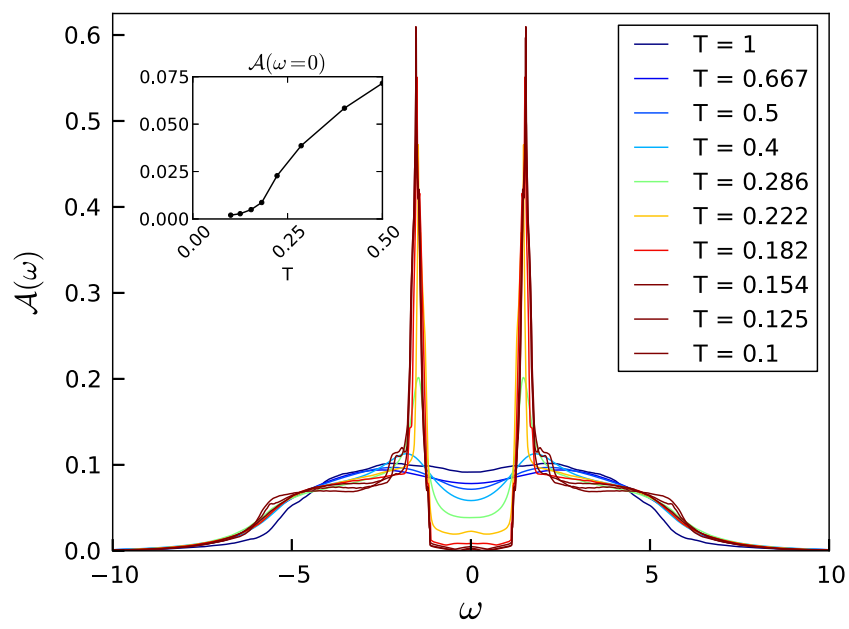

FIG. 5. (Color online) Temperature dependence of the spectrum $\mathcal{A}$ at half-filling. Notice the appearance of the van Hove singularities originating from the band splitting at the Fermi energy $\omega=0$. The standard deviation on the spectrum was extremely small in this case, since there is no Fermionic sign problem at half-filling.
Next we focus on the momentum anisotropy of the selfenergy and its impact on the spectrum. In Fig. 6, we show the imaginary part of the self-energy and the spectrum for three different $\vec{k}$ points along the Fermi surface. By looking at the rate of divergence on the imaginary axis of the self-energy and investigating the local densities, Werner et al. [25] argued that the spectrum at the Fermi energy on the antinodal points should disappear at a much faster rate then at the nodal points. The CPE confirms these findings. The imaginary part of the self-energy at the antinodal points is much larger than at the nodal points. Since the spectrum is inversely proportional to the self-energy, the spectrum vanishes faster at the antinodal points. Furthermore, we can immediately observe that this anisotropy in the self-energy increases as the temperature $T$ is lowered. Using the partial occupancies $n_{\vec{K}}$ in the different patches of the Brillouin zone, Gull et al. [27] have claimed that the gap opened at the nodal region should be much bigger than at the antinodal region. Defining the width of the gap as the minimum distance between the two van Hove singularities in Fig. 6, it can be seen that the claim of an anisotropic gap in the spectrum is also confirmed.

Finally, we discussed features that to our knowledge have not yet been reported in the literature. In particular, we would like to draw attention to the formation of the valleys in the imaginary part of the self-energy at $\omega \approx \pm 2$. Going from $T^{-1}=3$ to $T^{-1}=7$, we see that these valleys grow faster and are more profound at the antinodal points than at the nodal points. Since the imaginary part of the self-energy can be thought of as the inverse lifetime of the quasiparticle, we can conclude that the quasiparticle will have a short lifetime on the Fermi energy and a much longer one in the valleys. This picture translates directly into to the spectrum, where two peaks rapidly grow at $\omega \approx \pm 2$. The difference in the shape of these peaks can be explained by the topology of the free dispersion $\epsilon(\vec{k})$. The free dispersion is extremely flat at the antinodal points, since both the first derivative and the laplacian vanishes at this point. The spectral weight at the nodal point will thus be extremely peaked at the Fermi energy if no interaction is present. However, if there is a nonzero interaction, the lifetime of the particles at the Fermi energy will be very short, and all of them will be scattered in equal amounts to higher or lower energy-levels. At the nodal points, the free-dispersion spectrum is essentially linear with Fermi-velocity vector $\epsilon(\vec{k}) \sim \vec{k} \vec{v}_{F}$. Hence, the free spectrum will be smeared around the Fermi energy and have some spectral weight in the valleys of the imaginary part of the self-energy. Hence, these electrons will not be scattered away, when an interaction is introduced into the system. With this simple picture in mind, one can now easily understand shape difference of the peaks in the spectrum shown in Fig. 6, as well as the findings of Gull et al. with the partial occupancies as a function of the chemical potential.

\section{B. Electronic structure of $\mathrm{NiO}$}

We will now apply the CPE to a multiband model of $\mathrm{NiO}$ with materials-specific parameters derived from firstprinciples electronic structure calculations. $\mathrm{NiO}$ is a prototypical material with strong electronic correlations that has been 

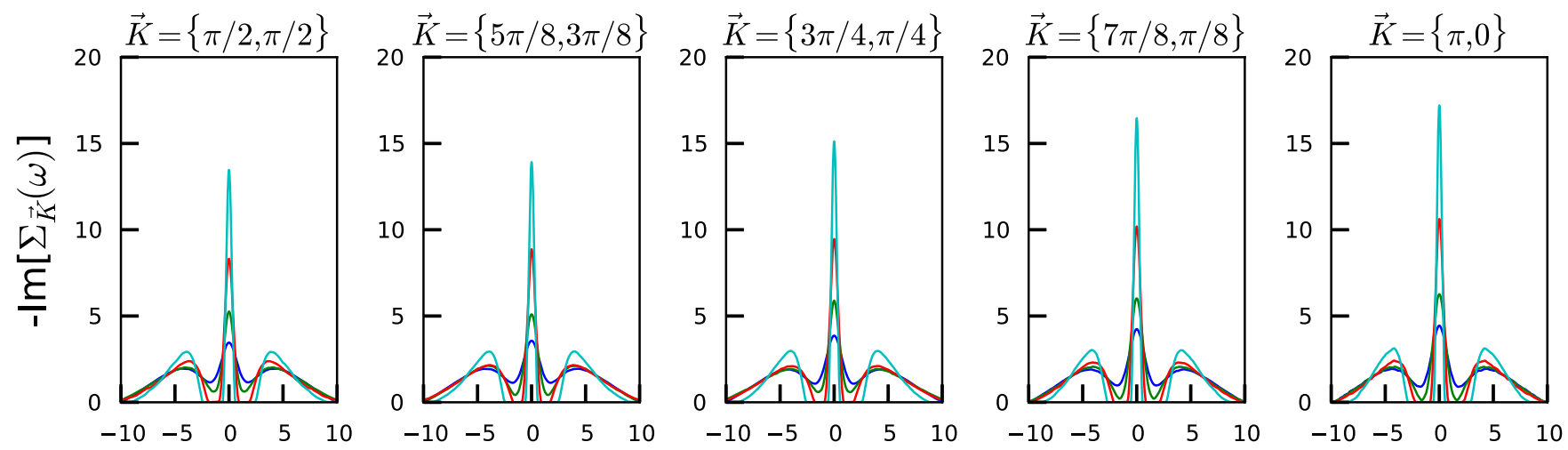

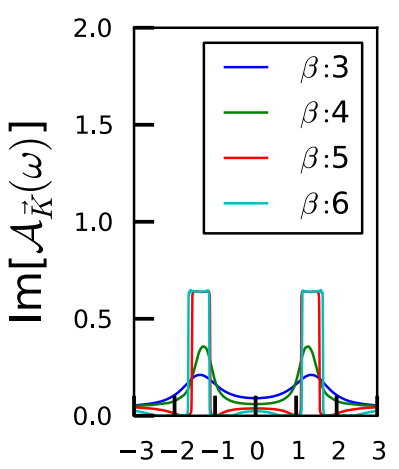

$\omega$

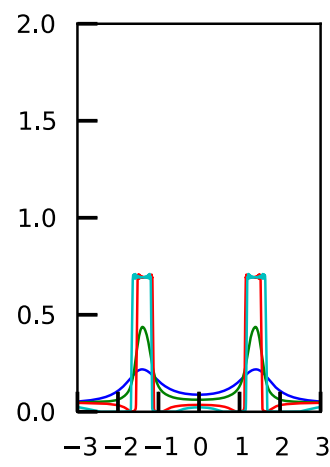

$\omega$

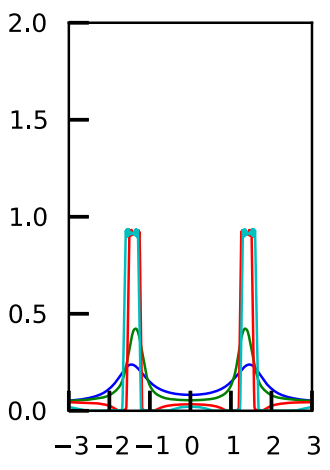

$\omega$

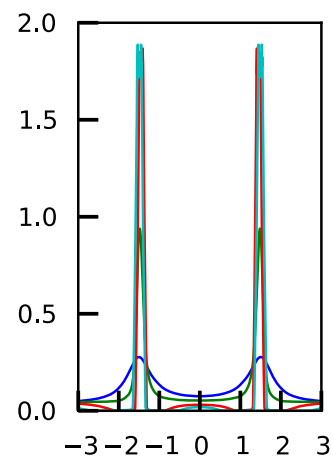

$\omega$

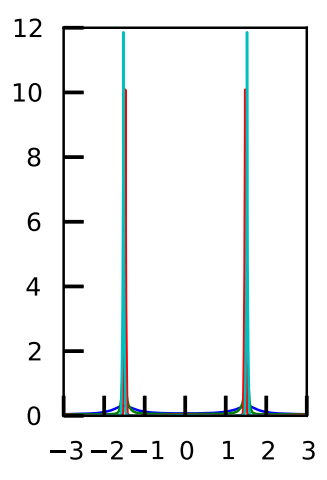

$\omega$

FIG. 6. (Color online) Temperature and momentum space dependence of the self-energy and spectrum along the Fermi surface in the single-band Hubbard model at half filling. The standard deviation on the self-energy and spectrum was extremely small in this case, since there is no Fermionic sign problem at half-filling.

extensively studied both experimentally [34-36] and theoretically [37-40]. The experimental results from the literature for this compound are the next best thing compared to the exact solution of the impurity model of a multiband system. It is thus an ideal testing ground for electronic structure calculations. Here we generate the Green's function and self-energies from LDA + DMFT-based Monte Carlo simulations [41,42], and subsequently apply the CPE for analytic continuation of the self-energy to obtain spectral function, which we compare directly to experiment.

The large insulating band gap of $4.3 \mathrm{eV}$ cannot be predicted by conventional band theory. Density functional theory within the local spin-density approximation (LSDA) [37] predicts $\mathrm{NiO}$ to be a band insulator, where the band gap is a consequence of the antiferromagnetic order. Angle-resolved photoemission experiments (ARPES), however, have shown [43] that the electronic band gap also exists in the paramagnetic phase, far above the Neel temperature at 525K. Furthermore, the predicted band gap with LSDA is considerably smaller than the experimental values. These deficiencies are corrected for by introducing correlation effects via DMFT. Here we will compare our calculated LDA + DMFT + CPE electronic spectra with experimental spectroscopy measurements in order to further validate the CPE method.

The impurity Hamiltonian $\mathcal{H}$ we study here is given in Eq. (27). It is of the usual $\mathrm{LDA}+\mathrm{U}$ form. In the present study, the LDA band structure of $\mathrm{NiO}$ was obtained by all-electron calculations using the linearized augmented plane wave (LAPW) method and is displayed in the inset of Fig. 7.
The resemblance of the band structure reported by Karolak et al. [42] is perfect and we can clearly observe the five nickel bands around the Fermi energy $\omega=0$ as well as the lower three oxygen bands around $\omega \approx-6$. The interaction tensor $U_{v, \sigma, \mu, \sigma^{\prime}}$ was obtained through a constrained RPA calculation (c-RPA) [44]. As is usual in the $\mathrm{NiO}$ compound, we have only kept the interaction terms between the nickel orbitals and ignore the density-density interaction between

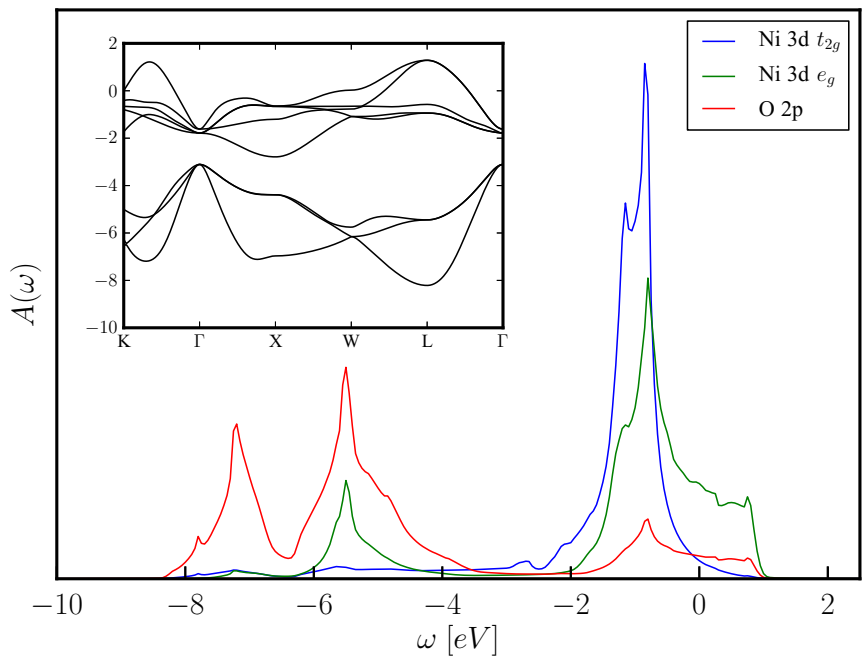

FIG. 7. (Color online) The noncorrelated partial density of states of $\mathrm{NiO}$ as obtained by LDA. Inset: band structure of $\mathrm{NiO}$ obtained with LDA. 
TABLE I. The interaction tensor $U_{v, \sigma, \mu, \sigma^{\prime}}$ for $\mathrm{NiO}$ obtained by c-RPA. The tensor can be approximated by the rotationally invariant Slater-Kanamori interaction matrix, using $U=9.14$ and $J=0.71$, which are close to the commonly accepted values of $U=8$ and $J=1$ in the literature.

\begin{tabular}{lccccc}
\hline \hline$U_{v, \sigma, \mu,-\sigma}$ & $n_{t_{2 g},-\sigma}$ & $n_{t_{2 g},-\sigma}$ & $n_{e_{g},-\sigma}$ & $n_{t_{2 g},-\sigma}$ & $n_{e_{g},-\sigma}$ \\
\hline$n_{t_{2 g}, \sigma}$ & 9.14 & 7.60 & 7.37 & 7.60 & 8.28 \\
$n_{t_{2 g}, \sigma}$ & 7.60 & 9.14 & 8.06 & 7.60 & 7.60 \\
$n_{e_{g}, \sigma}$ & 7.37 & 8.06 & 9.14 & 8.06 & 7.37 \\
$n_{t_{2 g}, \sigma}$ & 7.60 & 7.60 & 8.06 & 9.14 & 7.60 \\
$n_{e_{g}, \sigma}$ & 8.28 & 7.60 & 7.37 & 7.60 & 9.14 \\
$U_{v, \sigma, \mu, \sigma}$ & $n_{t_{2 g}, \sigma}$ & $n_{t_{2 g}, \sigma}$ & $n_{e_{g}, \sigma}$ & $n_{t_{2 g}, \sigma}$ & $n_{e_{g}, \sigma}$ \\
\hline$n_{t_{2 g}, \sigma}$ & 0.00 & 6.83 & 6.49 & 6.83 & 7.85 \\
$n_{t_{2 g}, \sigma}$ & 6.83 & 0.00 & 7.51 & 6.83 & 6.83 \\
$n_{e_{g}, \sigma}$ & 6.49 & 7.51 & 0.00 & 7.51 & 6.49 \\
$n_{t_{2 g}, \sigma}$ & 6.83 & 6.83 & 7.51 & 0.00 & 6.83 \\
$n_{e_{g}, \sigma}$ & 7.85 & 6.83 & 6.49 & 6.83 & 0.00 \\
\hline \hline
\end{tabular}

the oxygen-oxygen and oxygen-nickel orbitals. In the c-RPA method, the Wannier-orbitals, which are used to construct the tight-binding Hamiltonian $\mathcal{H}_{\mathrm{LDA}}$ of the impurity, are reused to construct the interaction tensor $U_{\nu, \sigma, \mu, \sigma^{\prime}}$. As a consequence, the interaction terms are more consistent with the band structure than a simple application of the rotationally invariant Slater-Kanamori $[45,46]$ onsite interaction matrix, which is traditionally constructed with the help of the parameters $U$ and $J$. However, a least-squares fit of the the c-RPA matrix toward the parameters $U$ and $J$ reveals that the interaction matrix can be approximated quite well with the parameters $U=9.14$ and $J=0.71$. These values do not differ tremendously from the original $U=8$ and $J=1$ parameters by Karolak. For the sake of completeness, we have listed the interaction tensor $U_{\nu, \sigma, \mu, \sigma^{\prime}}$ in Table I.

The multiband impurity problem in the self-consistent DMFT loop was solved by an implementation of the CT-HYB algorithm [11,12]. As is common in the literature [40], only the diagonal elements of the self-energy matrix are computed with the CT-HYB algorithm and the off-diagonal elements are ignored. Our calculations were performed at an inverse temperature of $\beta=5 \mathrm{eV}^{-1}$. At this temperature, the material is in the paramagnetic state and the correlations are strong enough to introduce a band gap. Since DMFT introduces correlations that have already been partly accounted for in the LDA functional, a double-counting correction $\mathcal{H}_{\text {dc }}$ needs to be applied to our impurity Hamiltonian $\mathcal{H}$ in Eq. (27). For this we follow the standard procedure of Karolak et al. [42]. It should be noted that the sum over the $m$ in the double-counting term $\mathcal{H}_{\mathrm{dc}}$ only runs over the nickel orbitals:

$$
\mathcal{H}=\mathcal{H}_{\mathrm{LDA}}-\underbrace{\mu_{d c} \sum_{m \sigma} n_{m \sigma}}_{\mathcal{H}_{\mathrm{dc}}}+\underbrace{\frac{1}{2} \sum_{\nu, \sigma, \mu, \sigma^{\prime}} U_{v, \sigma, \mu, \sigma^{\prime}} n_{v, \sigma} n_{\mu, \sigma^{\prime}}}_{\mathcal{H}_{\text {int }}} .
$$

No rigorous derivation is known for the double-counting term $\mathcal{H}_{\mathrm{dc}}$. Furthermore, the Mott-insulator gap increases with decreasing value of the the double-counting correction, and

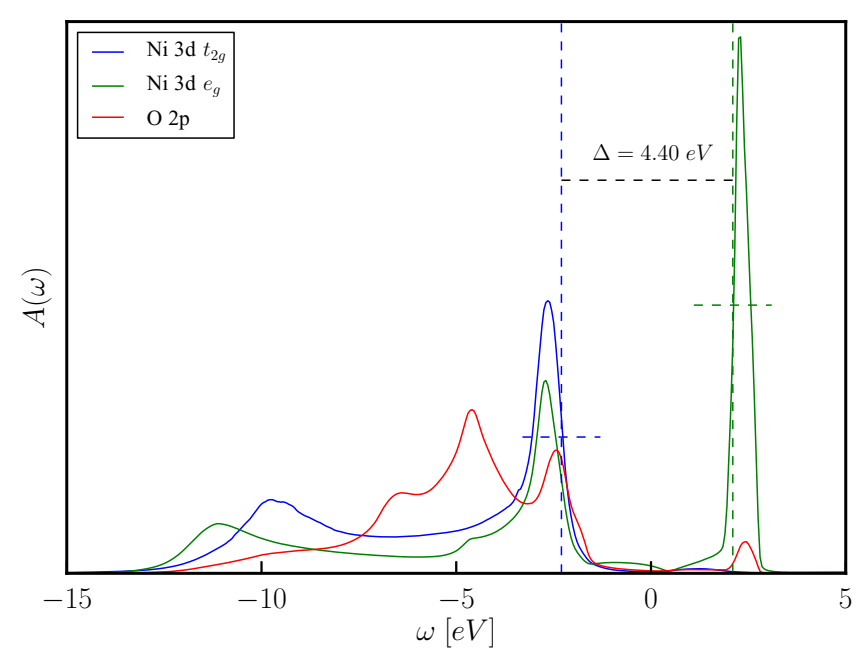

FIG. 8. (Color online) The correlated partial spectra of $\mathrm{NiO}$ calculated by LDA + DMFT + CPE. Defining the band gap as in experimental physics, i.e., the distance between the midpoints of the top of the peaks, we obtain a band gap of $4.4 \mathrm{eV}$, in good agreement with the experimental value of $4.3 \mathrm{eV}$.

the parameter $\mu_{\mathrm{dc}}$ is thus tuned to fit experiment. Since we are only interested in validating the CPE algorithm, we apply the commonly used value $\mu_{\mathrm{dc}}=25 \mathrm{eV}$ and test wether our method indeed reproduced a bad gap that is in agreement with the expected value of $4.3 \mathrm{eV}$.

Once a density-density interaction is included between the nickel orbitals by means of a self-consistent DMFT calculation, a band gap appears in the spectral density. This can be clearly seen in Fig. 8, where the partial spectra of each orbital are displayed. As usual, Fig. 8 was obtained by performing an analytical continuation of the Matsubara self energy to the real axis with an offset of $\delta=0.1$. A consecutive tetrahedron integration over the entire Brillouin zone then results in the lattice Green's function, from which the partial spectrum $A_{v}(\omega)$ can be obtained. If we use the same definition of the band gap from experimental physics, i.e., the distance between the midpoints of the top of the peaks, we obtain a band gap of $4.4 \mathrm{eV}$, in good agreement with the experimental value of $4.3 \mathrm{eV}$ obtained by Sawatsky and Allen [35]. These midpoints are represented by the horizontal dotted lines in Fig. 8. Their intersection with the peak is marked by the vertical dotted lines, which difference defines the band gap.

From the comparison between the ED results and CPE in Sec. IV, we know that the CPE is very good at reproducing an accurate picture for the spectrum close to the Fermi energy. As such, it is not surprising to reproduce the correct band gap of $\mathrm{NiO}$ around the Fermi energy. To further benchmark the CPE, we will compare the calculated spectrum with the experimental spectrum obtained by Sawatzky and Allen [35]. In this way, we want to explore how the CPE behaves over the entire real axis and whether it can capture the essential physics far from the Fermi energy, as we claimed in the exact diagonalization section. The experimental spectrum was obtained as a combination of XPS and BIS measurements on cleaved single crystals of $\mathrm{NiO}$. The XPS spectrum was recorded at $120 \mathrm{eV}$ and mainly captures the nickel 3D character. The measured spectra is shown in Fig. 9, together with the 


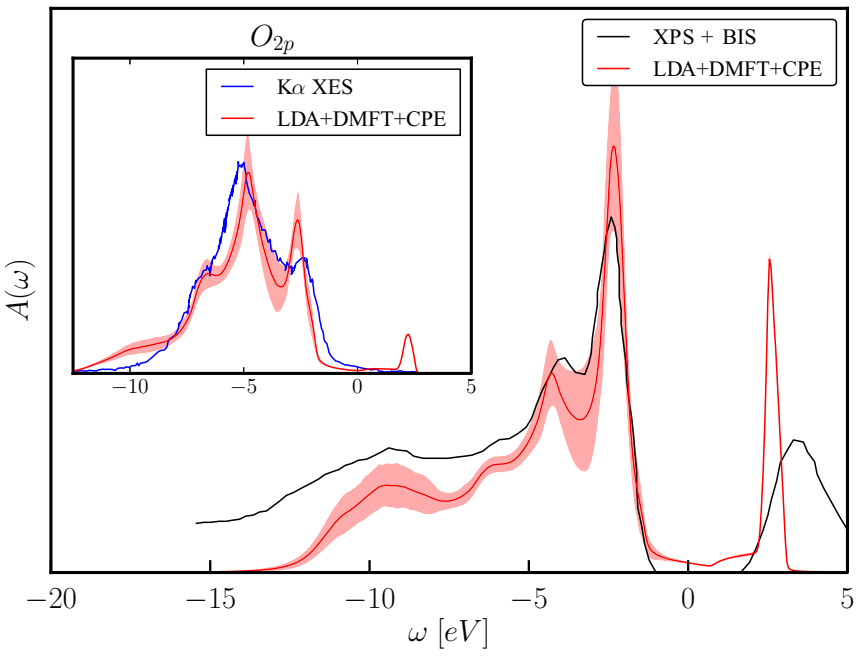

FIG. 9. (Color online) The comparison of XPS and BIS spectra [35] to the spectral function of NiO calculated by LDA + DMFT + $\mathrm{CPE}$. The XPS spectrum is measured at $120 \mathrm{eV}$, showing primarily the nickel 3D character. Inset: The comparison of $\mathrm{K} \alpha$-emission spectra [47] with the partial spectrum of the $O_{2 p}$ orbital calculated by LDA + DMFT + CPE.

$\mathrm{LDA}+\mathrm{DMFT}+\mathrm{CPE}$ spectrum. The latter is obtained by summing up the partial spectra depicted in Fig. 8 and multiplied with the multiplicity of each orbital $\left(3 \times t_{2 g}, 2 \times\right.$ $e_{g}, 3 \times O_{2 p}$ ). Since the spectroscopy is measured in arbitrary units of intensity, we can scale the measured spectrum such that the largest peaks have the same height. A simple comparison of both spectra shows a very good agreement between measured and computed spectrum. In the region $[-15,-5]$, we can observe that the CPE gradually rises and appears to reproduce some of the peaks, albeit with a slight left shift of approximately $2 \mathrm{eV}$. The peaks that define the gap around the Fermi surface are also much sharper defined with the CPE.

To further validate the CPE, we compare the partial spectrum of the $O_{2 p}$ orbitals. The latter was measured very accurately by Kurmaev et al. [47] with x-ray emission spectroscopy (XES). The oxygen $K$-edge emission spectrum provides a representation of the $O_{2 p}$ spectrum and can thus be readily used to compare with the calculated $O_{2 p}$ spectrum. In the inset of Fig. 9, we compare the measured with the computed partial spectrum. Just as with the total spectrum, we can observe a very good agreement between theory and experiment and much sharper peaks and valleys in the CPE. The figure also shows that the CPE can describe the essential physics far away from the Fermi surface rather well, as claimed in the ED section. This is not surprising, since the CPE produces a smooth self-energy on the real axis, which is consistent with the basic assumption of a mean field theory such as the DMFT.

\section{SUMMARY AND CONCLUSIONS}

We have presented a new algorithm, the CPE, to analytically continue the self-energy of quantum many-body systems from (complex) Matsubara frequencies to the real axis. This method allows straightforward computation of electronic spectra for lattice models of strongly correlated systems from self-energy data collected in DMFT simulations. The need for such an algorithm arises from developments of new, efficient continuoustime quantum Monte Carole solvers for DMFT, which, in conjunction with nonequidistant fast fourier transform, allow direct accumulation of the Green's function and self-energy on the Matsubara frequencies with controlled accuracy.

Since analytical continuation of complex functions is notoriously unstable, we have developed the CPE algorithm on the basis of two well-known analytic properties of the self-energy: (1) its imaginary part has a branch cut along the real axis and is negative definite in the upper complex plane (i.e., $\left.\lim _{\varpi \rightarrow 0} \operatorname{Im}[\Sigma(\vec{k}, \omega+\imath \varpi)] \leqslant 0\right)$; and (2) it is analytic and has no poles in the upper complex plane. Thus, as a consequence of the first property the imaginary part of the self-energy can be parametrized as a purely negative function, which is a strong constraint, and the analyticity allows use of the Kramers-Kronig relationship to compute the self-energy everywhere in the complex plane from the imaginary part on the real axis. One can hence compute the self-energy on the Matsubara frequencies for any given parametrization of the imaginary part on the real axis. With this in mind, the CPE algorithm can be summarized in one sentence: It consists of finding a negative definite parametrization of the imaginary part of the self-energy on the real frequency axis in such as way that the difference between accumulated QMC self-energy (data) and the computed self-energy on the Matsubara frequencies is minimized.

With this the analytical continuation problem has been cast into a contained minimization problem. Minimization is much more stable numerically, especially with regard to statistical noise in the data that arises from the Monte Carlo sampling. Moreover, in the present case, the constrained minimization can be formulated as a quadratic programmable optimization with linear constraints. The latter is a well-known problem for which many numerical algorithms exist. In the present paper we have used the simplest, the Frank-Wolfe algorithm.

Extensive validation of the $\mathrm{CPE}$ algorithm has been given in this paper, both in terms of exactly solvable finite-size models as well as for lattice problems that are well known in the literature. The strengths and weaknesses of the CPE have been analyzed in terms of a direct comparison with exact solutions for an isolated eight-site cluster. The CPE reproduces very well the features of the spectral function near the Fermi energy. Farther away from the Fermi level, the CPE only reproduces broad features and is unable to track the sharp features of the spectral functions that result from individual eigenvalues of the Green's function matrix of the finite-size system.

The first of two lattice problems used for the validation of the CPE algorithm is the 2D Hubbard model at half-filling, and in particular the momentum-dependent gap formation for which many results based on the identity in Eq. (25) have been published recently. This identity has been validated with the CPE over a wide temperature range, and our results agree with the literature. Furthermore, the CPE allowed us to conclude from the $k$-dependent spectrum that the gap is indeed smaller at the nodal than at the antinodal points.

Finally, the CPE was used to compute the spectrum of $\mathrm{NiO}$, a prototypical strongly correlated materials that has been extensively studied in experiment and simulation. The standard method of LDA+DMFT to compute the self-energy 
has been applied and the spectra computed with the CPE algorithm are in excellent agreement with XPS, XES, and BIS measurements on $\mathrm{NiO}$ published in the literature (see Fig. 9). This demonstrates that the CPE can be used as a robust, unambiguous method to compete spectral function of real materials from self-energy data that has been collected on the Matsubara frequencies in effective medium-based quantum Monte Carlo simulations.
[1] A. Georges, G. Kotliar, W. Krauth, and M. J. Rozenberg, Rev. Mod. Phys. 68, 13 (1996).

[2] H. Vidberg and J. Serene, J. Low Temp. Phys. 29, 179 (1977).

[3] J. E. Gubernatis, M. Jarrell, R. N. Silver, and D. S. Sivia, Phys. Rev. B 44, 6011 (1991).

[4] M. Jarrell and J. E. Gubernatis, Phys. Rep. 269, 133 (1996).

[5] R. N. Silver, D. S. Sivia, and J. E. Gubernatis, Phys. Rev. B 41, 2380 (1990).

[6] H. B. Schüttler and D. J. Scalapino, Phys. Rev. Lett. 55, 1204 (1985).

[7] H. B. Schüttler and D. J. Scalapino, Phys. Rev. B 34, 4744 (1986).

[8] C. E. Shannon and W. Weaver, Bell Syst. Tech. J. 27, 379 (1948).

[9] A. W. Sandvik, Phys. Rev. B 57, 10287 (1998).

[10] A. N. Rubtsov, V. V. Savkin, and A. I. Lichtenstein, Phys. Rev. B 72, 035122 (2005).

[11] P. Werner, A. Comanac, L. de' Medici, M. Troyer, and A. J. Millis, Phys. Rev. Lett. 97, 076405 (2006).

[12] P. Werner and A. J. Millis, Phys. Rev. B 74, 155107 (2006).

[13] E. Gull, P. Werner, O. Parcollet, and M. Troyer, Europhys. Lett. 82, 57003 (2008).

[14] E. Gull, A. J. Millis, A. I. Lichtenstein, A. N. Rubtsov, M. Troyer, and P. Werner, Rev. Mod. Phys. 83, 349 (2011).

[15] E. Gull, P. Staar, S. Fuchs, P. Nukala, M. S. Summers, T. Pruschke, T. C. Schulthess, and T. Maier, Phys. Rev. B 83, 075122 (2011).

[16] P. Staar, T. A. Maier, M. S. Summers, G. Fourestey, R. Solca, and T. C. Schulthess, in Proceedings of SC13: International Conference for High Performance Computing, Networking, Storage and Analysis, SC 2013 (ACM, New York, NY, USA, 2013), pp. 1:1-1:11.

[17] P. Staar, T. A. Maier, and T. C. Schulthess, J. Phys.: Conf. Ser. 402, 012015 (2012).

[18] X. Wang, E. Gull, L. de' Medici, M. Capone, and A. J. Millis, Phys. Rev. B 80, 045101 (2009).

[19] A. A. Abrikosov, L. P. Gorkov, and I. Y. Dzyaloshinskii, Quantum Field Theoretical Methods in Statistical Physics, International Series of Monographs in Natural Philosophy, Vol. 4 (Pergamon Press, Oxford, 1965).

[20] J. Hubbard, Proc. R. Soc. London A 276, 238 (1963).

[21] M. H. Hettler, A. N. Tahvildar-Zadeh, M. Jarrell, T. Pruschke, and H. R. Krishnamurthy, Phys. Rev. B 58, R7475 (1998).

[22] M. H. Hettler, M. Mukherjee, M. Jarrell, and H. R. Krishnamurthy, Phys. Rev. B 61, 12739 (2000).

[23] M. Jarrell, T. Maier, C. Huscroft, and S. Moukouri, Phys. Rev. B 64, 195130 (2001).

[24] T. Maier, M. Jarrell, T. Pruschke, and M. H. Hettler, Rev. Mod. Phys. 77, 1027 (2005).

[25] P. Werner, E. Gull, O. Parcollet, and A. J. Millis, Phys. Rev. B 80, 045120 (2009).

[26] E. Gull, O. Parcollet, P. Werner, and A. J. Millis, Phys. Rev. B 80, 245102 (2009).
[27] E. Gull, M. Ferrero, O. Parcollet, A. Georges, and A. J. Millis, Phys. Rev. B 82, 155101 (2010).

[28] We refer here to the interior uniqueness properties of single-valued complex functions from http://www. encyclopediaofmath.org:Let $\mathcal{D}$ be a domain in the complex plane $\mathbb{C}$. The classical interior uniqueness theorem for holomorphic, i.e., single-valued analytic functions on $\mathcal{D}$ states that if two holomorphic functions $f(z)$ and $g(z)$ in $\mathcal{D}$ coincide on some set $\mathcal{E} \subset \mathcal{D}$ containing at least one limit point in $\mathcal{D}$, then $f(z)=g(z)$ everywhere in $\mathcal{D}$.

[29] M. Frank and P. Wolfe, Naval Res. Logistics Quart. 3, 95 (1956).

[30] http://mathworld.wolfram.com/RampFunction.html.

[31] E. Anderson, Z. Bai, J. Dongarra, A. Greenbaum, A. McKenney, J. Du Croz, S. Hammerling, J. Demmel, C. Bischof, and D. Sorensen, in Proceedings of the 1990 ACM/IEEE conference on Supercomputing, Supercomputing 1990 (IEEE Computer Society Press, Los Alamitos, CA, USA, 1990), pp. 2-11.

[32] P. Lambin and J. P. Vigneron, Phys. Rev. B 29, 3430 (1984).

[33] J.-H. Lee, T. Shishidou, and A. J. Freeman, Phys. Rev. B 66, 233102 (2002).

[34] J. M. McKay and V. E. Henrich, Phys. Rev. Lett. 53, 2343 (1984).

[35] G. A. Sawatzky and J. W. Allen, Phys. Rev. Lett. 53, 2339 (1984).

[36] Z.-X. Shen, R. S. List, D. S. Dessau, B. O. Wells, O. Jepsen, A. J. Arko, R. Barttlet, C. K. Shih, F. Parmigiani, J. C. Huang, and P. A. P. Lindberg, Phys. Rev. B 44, 3604 (1991).

[37] K. Terakura, T. Oguchi, A. R. Williams, and J. Kübler, Phys. Rev. B 30, 4734 (1984).

[38] X. Ren, I. Leonov, G. Keller, M. Kollar, I. Nekrasov, and D. Vollhardt, Phys. Rev. B 74, 195114 (2006).

[39] J. Kunes, V. I. Anisimov, A. V. Lukoyanov, and D. Vollhardt, Phys. Rev. B 75, 165115 (2007).

[40] J. Kunes, V. I. Anisimov, S. L. Skornyakov, A. V. Lukoyanov, and D. Vollhardt, Phys. Rev. Lett. 99, 156404 (2007).

[41] G. Kotliar, S. Y. Savrasov, K. Haule, V. S. Oudovenko, O. Parcollet, and C. A. Marianetti, Rev. Mod. Phys. 78, 865 (2006).

[42] M. Karolak, G. Ulm, T. Wehling, V. Mazurenko, A. Poteryaev, and A. Lichtenstein, J. Electron Spectrosc. Relat. Phenom. 181, 11 (2010).

[43] O. Tjernberg, S. Söderholm, G. Chiaia, R. Girard, U. O. Karlsson, H. Nylén, and I. Lindau, Phys. Rev. B 54, 10245 (1996).

[44] A. Kozhevnikov, A. G. Eguiluz, and T. C. Schulthess, in Proceedings of the 2010 ACM/IEEE International Conference for High Performance Computing, Networking, Storage and Analysis, SC 2010 (IEEE Computer Society, Washington, DC, USA, 2010), pp. 1-10.

[45] J. Kanamori, Prog. Theor. Phys. 30, 275 (1963).

[46] J. C. Slater, Phys. Rev. 49, 537 (1936).

[47] E. Z. Kurmaev, R. G. Wilks, A. Moewes, L. D. Finkelstein, S. N. Shamin, and J. Kunes, Phys. Rev. B 77, 165127 (2008). 